\title{
Protée
}

\section{Paul Lussier • Fragments}

\section{Louise Déry}

Volume 30, numéro 3, hiver 2002

Autour de Peirce : poésie et clinique

URI : https://id.erudit.org/iderudit/006868ar

DOI : https://doi.org/10.7202/006868ar

Aller au sommaire du numéro

Éditeur(s)

Département des arts et lettres - Université du Québec à Chicoutimi

ISSN

0300-3523 (imprimé)

1708-2307 (numérique)

Découvrir la revue

Citer cet article

Déry, L. (2002). Paul Lussier • Fragments. Protée, 30(3), 55-62.

https://doi.org/10.7202/006868ar d'utilisation que vous pouvez consulter en ligne.

https://apropos.erudit.org/fr/usagers/politique-dutilisation/ 


\section{PAUL LUSSIER • FRAGMENTS}
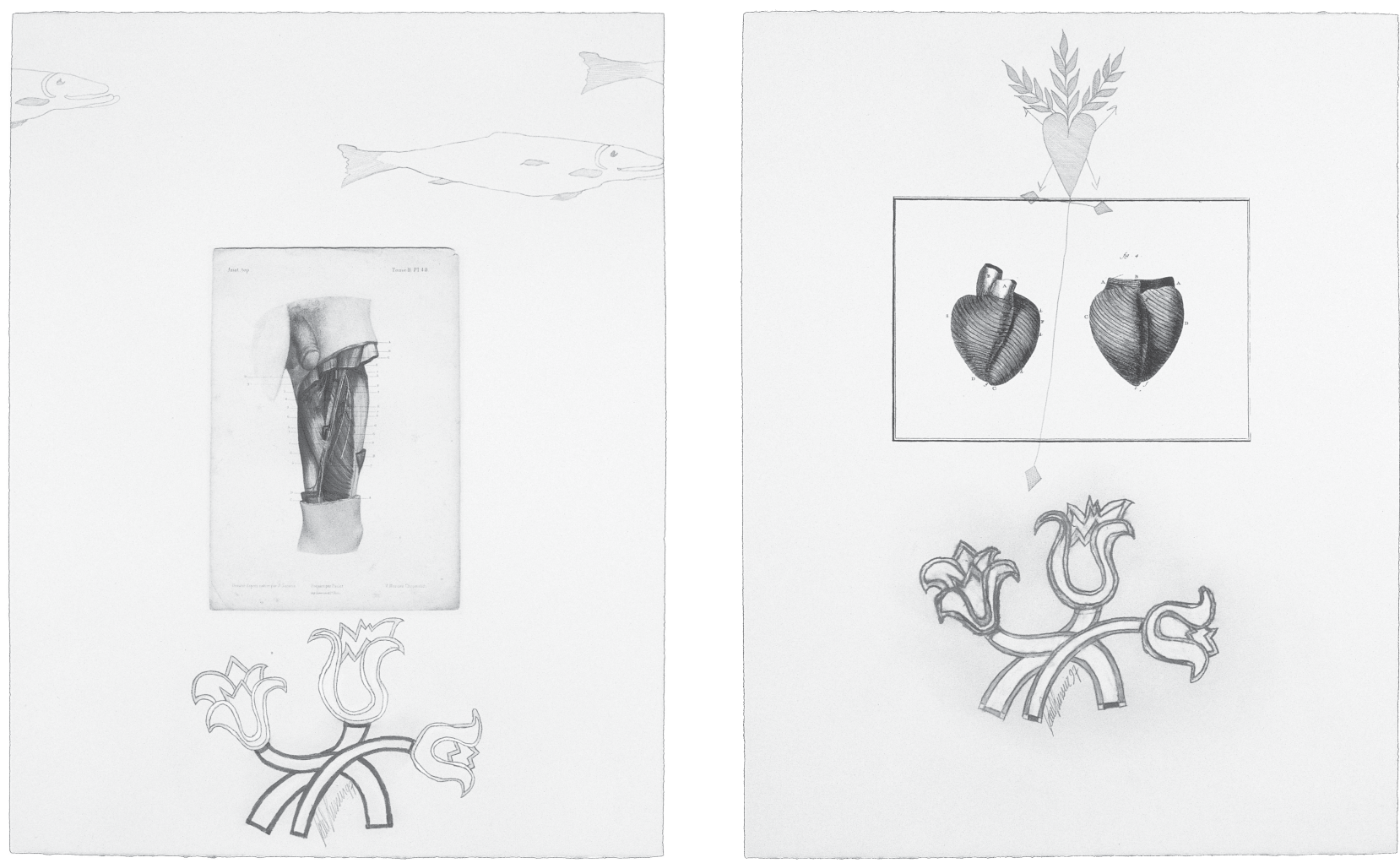

\section{PAR LOUISE DÉRY}

C'est en parlant d'une attention au détail que Paul Lussier propose spontanément une médiation de sa pratique. Dans un court texte de 1997 intitulé Détails. Aspects de la vie et de la mort de saint Sébastien racontés par le détail, il insiste sur ce qu'il voit comme un paradoxe entre la qualité de précision généralement attachée au détail et sa possible futilité quand il s'agit de qualifier une chose sans importance. Mais, en fin de propos, il utilise plutôt le mot fragment, pour référer aux restes de l'œuvre, pour en suggérer le deuil, pour ne retenir que l'essentiel.

Ainsi, Paul Lussier produit non pas une accumulation de signes, il pratique plutôt le désencombrement. Il ne s'agit pas d'un retrait, mais bien d'une opération qui se fait par soustraction, quand les signes, les figures et les volumes dessinés semblent les survivants d'une opération sélective. Réchappés. Rescapés. Si, dans le fait d'écrire, le texte pratique la langue, ici, sur le corps de la page, le dessin travaille la pensée. Mais il s'agit d'une pensée qui se garde des récits bien résolus, qui se montre hésitante à conclure quoi que ce soit, qui mise 
au contraire sur le fracas des questions, qui fait la vie dure aux propos de l'œuvre. Petites ruines, restes de texte en palimpseste, troubles apparents d'un monde disséqué où il convient de porter attention au cœur pour s'assurer qu'il bat, les feuilles dessinées de Paul Lussier ralentissent le fragment. Le fragment est lent, dit le poète Normand de Bellefeuille, cette idée me plaît. Cette idée me retient.

Paul Lussier pratique le dessin et la gravure depuis des années. Techniques et métiers de I'un et de l'autre appellent instantanément l'idée d'un travail qui s'accomplit dans la durée. Cette durée est propice à la saisie du temps en divers registres où alternent l'action, I'attente, I'hésitation, la reprise, le repentir... la fin. À moins que cela ne soit l'abandon. Lorsqu'il parle de deuil, cela n'empêche en rien l'artiste de donner vie à la technique, à la facture, à la touche. Car ses dessins sont animés par plusieurs effets de matière, ils sont marqués par la sensualité. Cela produit des pensées-images, des raccourcis, ou des fragments qui travaillent non pas la reconstitution de l'image mais sa déconstruction. Nous sommes ainsi en face de vestiges, pourtant il n'est aucunement question de nostalgie envers le manque, envers le manquement. Nous serions plutôt en face de l'image manquante, celle-là qui fait que la pensée est travaillée par l'espoir, que la forme se risque, que le texte n'est pas complètement oblitéré, que la voix ne se perd pas. Nous serions plutôt en face de l'image fragile, instable, incertaine. Nous serions en face de l'image fractionnée, répétée, réverbérée.

Parce qu'il enseigne, Paul Lussier est aussi une voix. Entre dire et faire, entre la promesse et l'aveu, entre la classe et l'atelier, seul ou entouré, I'artiste soigne ses « conduites créatrices », une expression de Paul Valéry qu'il aime faire sienne. Cela se fait aussi dans la durée, dans la répétition incessante de fragments de temps ralentis par l'image.

Les œuvres de Paul Lussier, reproduites dans ce numéro de Protée, sont des détails tirés de la suite de dessins de 1997 qui ont pour titre: Détails. Aspects de la vie et de la mort de saint Sébastien racontés par le détail. Ce sont des estampes numériques sur papier «Arches », format raisin $(50 \times 65 \mathrm{~cm})$, rehaussées au pastel, à la plombagine et au crayon de couleur. 


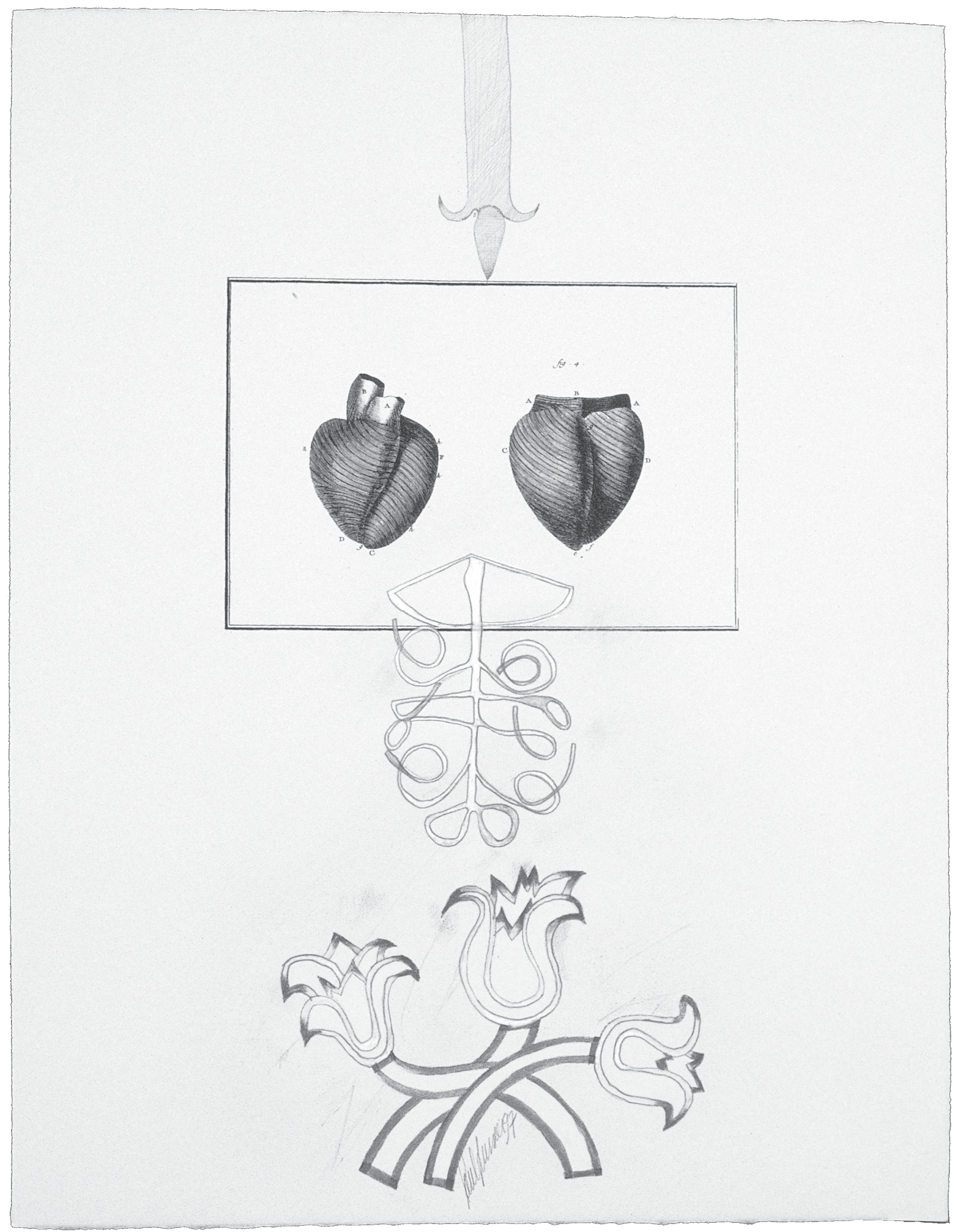




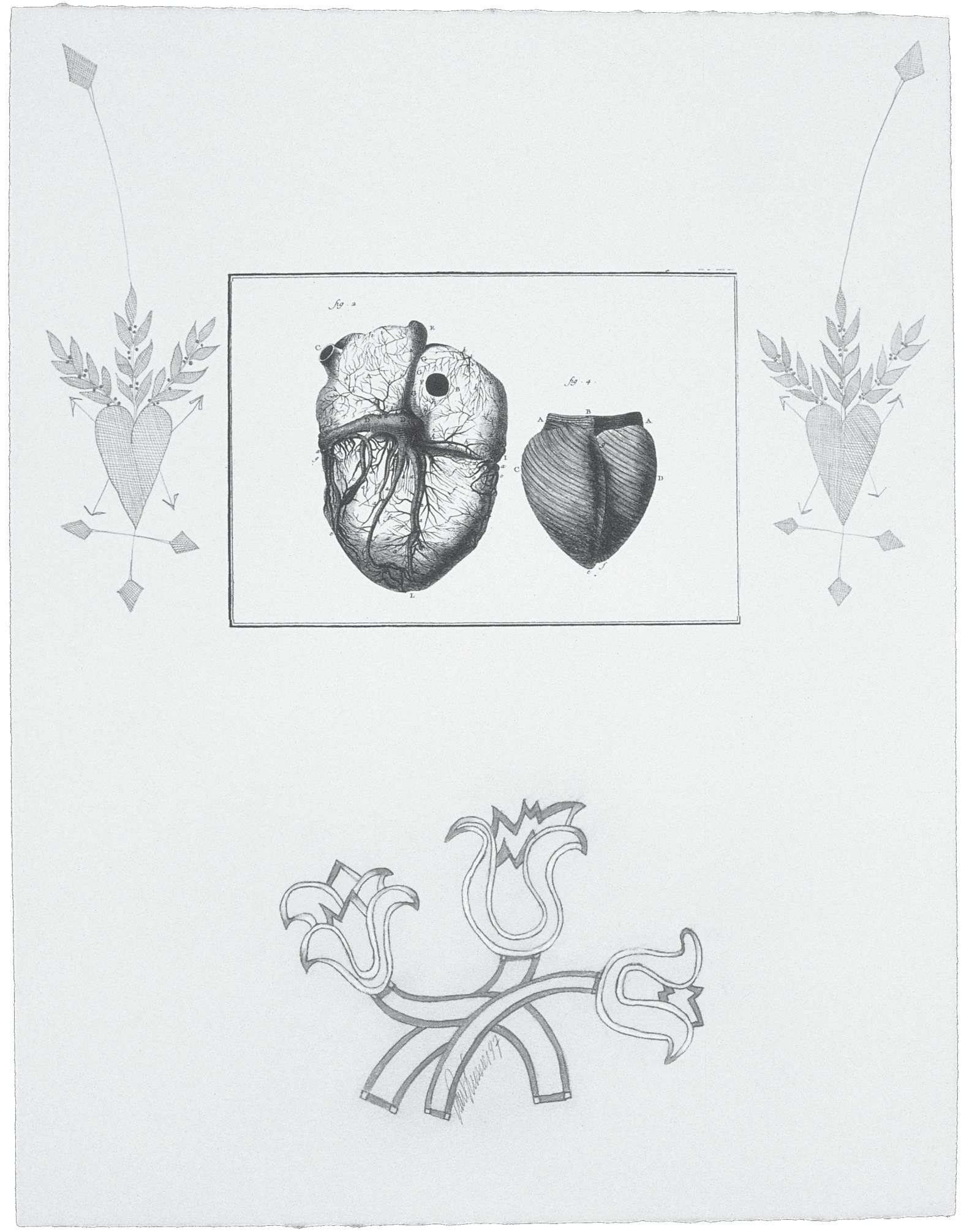




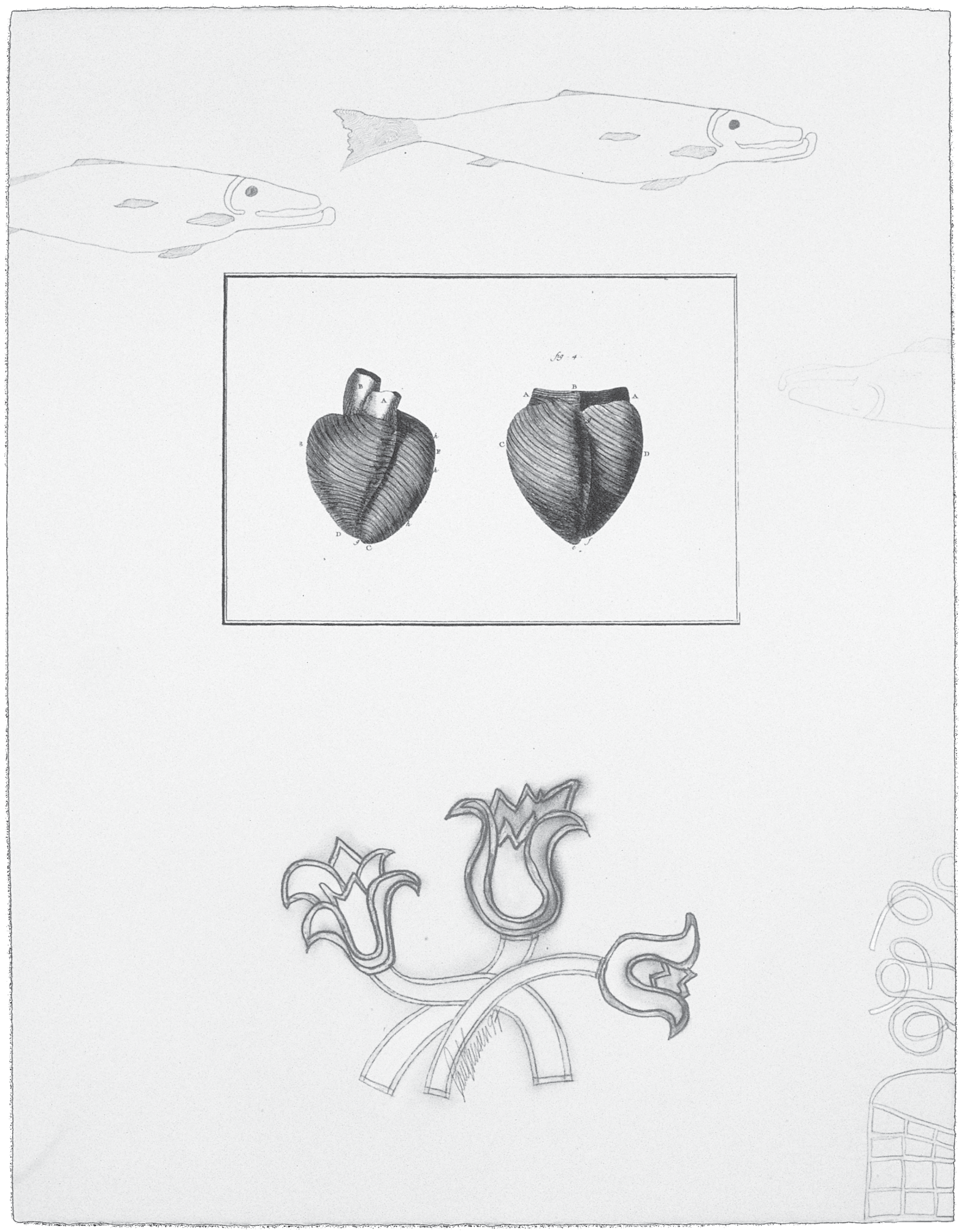




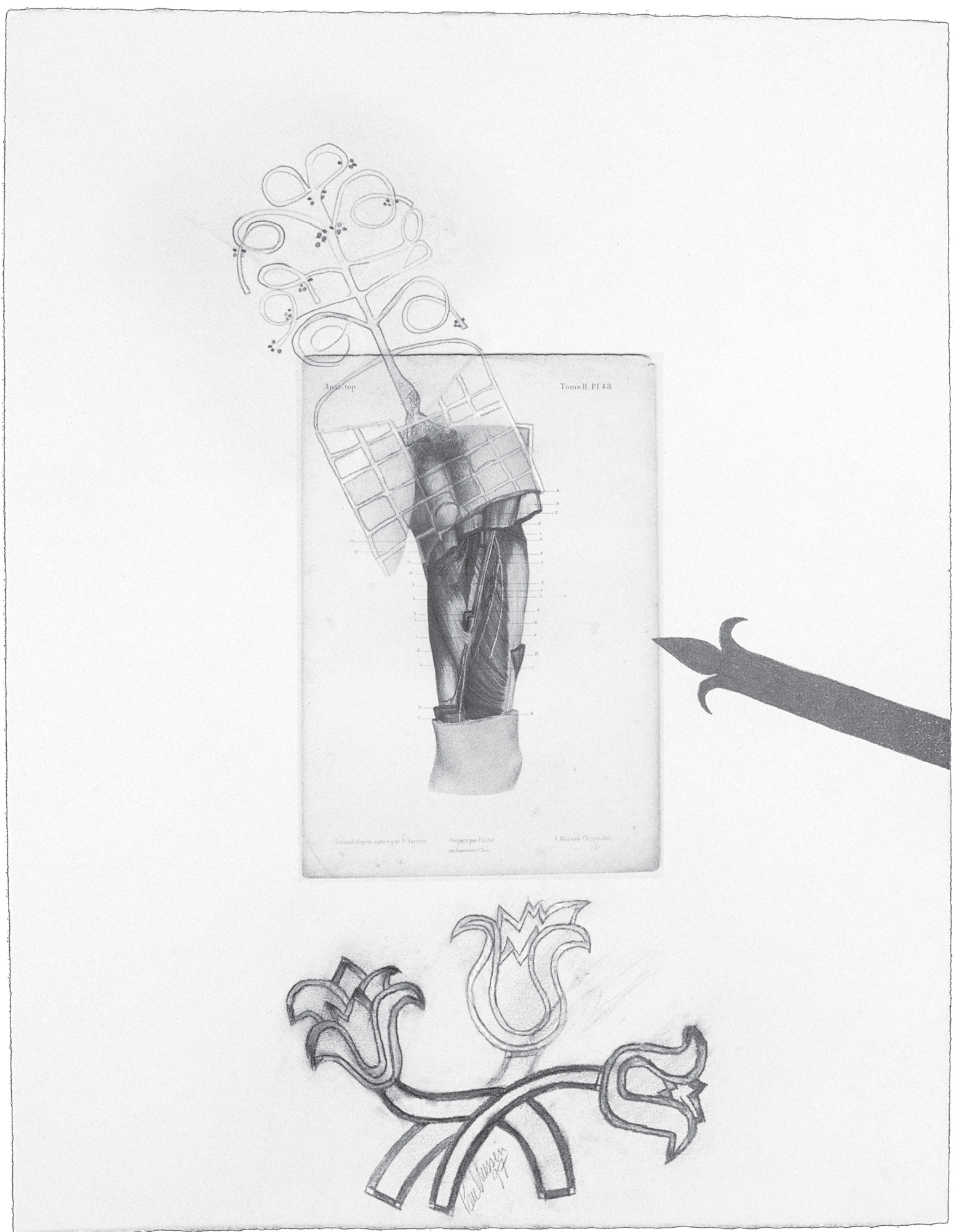




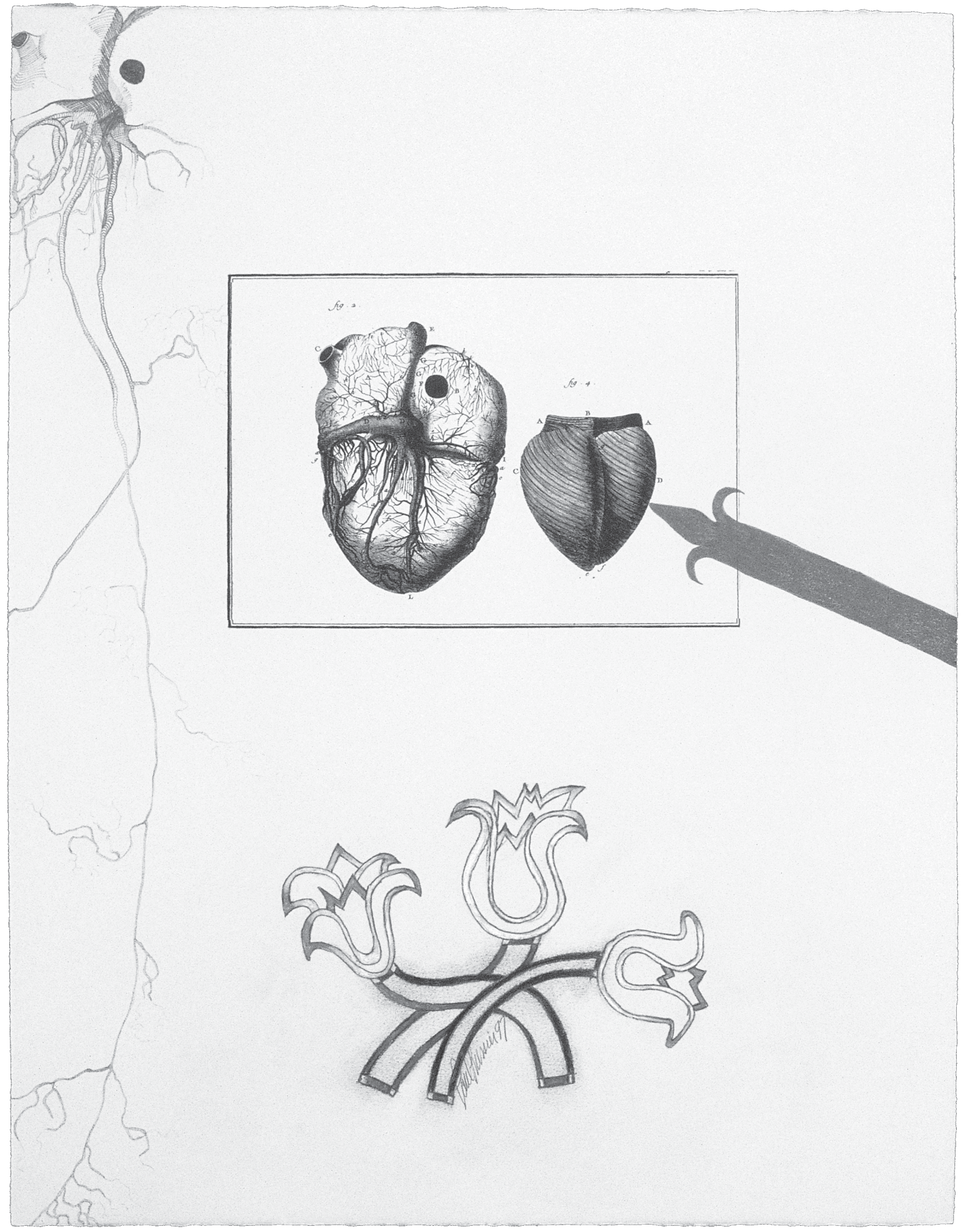




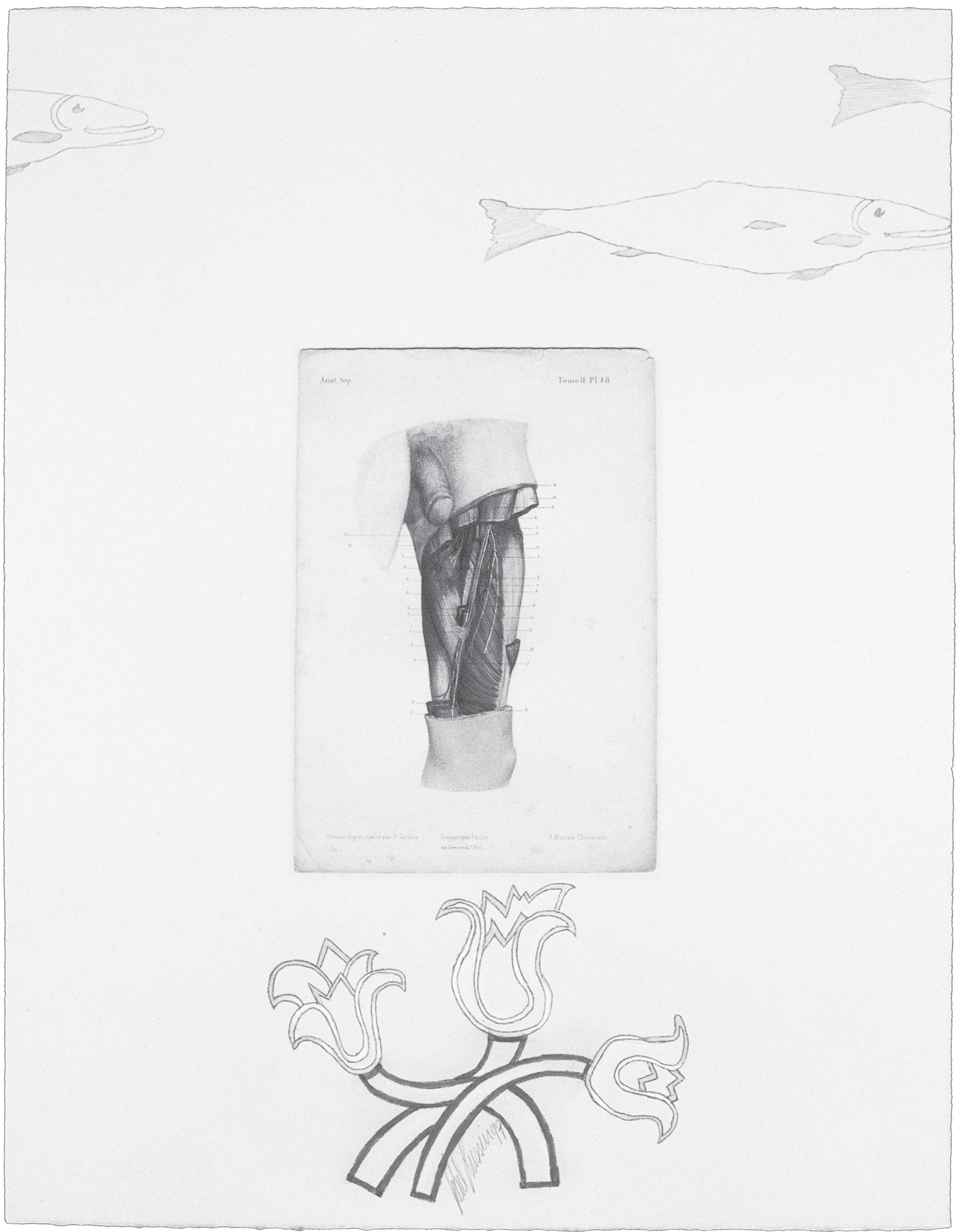

